\title{
Machine learning models to predict the precise progression of Tay-Sachs and Related Disease
}

\author{
Akanksha Limaye $^{1}$, Anuraj Nayarisseri ${ }^{1,2, *}$ \\ *Corresponding author: anuraj@eminentbio.com, Tel : +91 9752295342
}

1. In silico Research Laboratory, Eminent Biosciences, Mahalakshmi Nagar, Indore 452010,Madhya Pradesh, India.

2. Bioinformatics Research Laboratory, LeGene Biosciences Pvt Ltd., Mahalakshmi Nagar, Indore 452010, Madhya Pradesh, India.

\section{Background}

Tay Sachs is a very rare neurodegenerative disorder and the second most common lipid storage disorder. The testimony of TSD in infants is marked by the gradual loss in vision, hearing impairment, increased muscle stiffness and cherry red retinal spot. These symptoms progress to paralysis, dementia, seizures, neuro-regression leading to the demise of the patient by the age of 3 to 4 years of age. The neurological and visceral accumulation of glycosphingolipids accounts for the major morbidity and mortality of the patients [1]. Tay Sachs disorder (TSD) is characterised by the accumulation of the ganglioside in the brain nerve cells. The abnormal accumulation of the shpingolipids (GalNAc- \&1,4-(NeuNAc- \&2,3) -Gal+1,4-Glc_13-1,1\{2-N-acyl) sphingosine and GalNAc-/3-1,4-Gal_P1,4- GlcQ- 1,1(2-Nacyl)sphingosine) in nerve tissue leads to progressive dysfunction of the central nervous system [2]. When the enzyme 3-N-acetyl hexosaminidase (hexosaminidase A (HEXA)) is responsible for the breakdown of the fats- are defective, the glycosphingolipids increase excessively.[3] The HEXA gene, responsible for coding of the hexosaminidase A, is located on Chromosome 15. Currently, there are no promising cure for TSD as the treatment targets the symptoms of the disease [4]. The advanced techniques have introduced CRISPR/cas9 as the suggestive treatment plan.

NGS (next generation sequencing) is one such diagnostic tool- providing rapid and accurate genomic information of the patient. The speculation of CRISPR/Cas9 requires critical understanding of the key genes involved, in order to design the treatment plan. The CRISPR (clustered regularly interspaced repeats) technology allows scientists to make the precise change in the genetic code. Cas9 endonucleases is the associated protein which is guided through specifically designed guide-RNA to the target DNA. CRISPR/Cas9 selectively target genes containing mutations that lead to non-functional products and correct the diseasecausing mutations, in vitro and in vivo [5]. Currently the platforms used for gene editing for TSD patients include zinc finger nucleases (ZFNs), transcription activator-like effector nucleases (TALENs), and endonucleases. But these alternative platforms fail to provide ease and diversity in the areas where the CRISPR/cas9 excels. Owing to its ability to manipulate the target genes, however, the efficacy of the single guided RNA can be challenged, resulting in the unintended cleaving of the non-target sites. Thus, with the framework of the machine learning, using the algorithm of CRISTA the propensity of the cleaving the target site can be determined easily. The advanced techniques of machine learning along with NGS, provide varied scope of precise qualitative and quantitative study of the disorder. 
The suggested research work holds promising and revolutionary grounds in designing the treatment plan for the patient. The precise treatment can be planned on the basis of early diagnosis of the disease. The development of machine learning techniques in this field can assure a definite neurotherapeutics as the models can predict the precise progression of disease. The proposed work not only provides the research in the area of Tay Sachs, but also pose to cure neurodegenerative disorders such as Alzheimer's, dementia, Huntington's, etc which still remain mystery to the scientists.

\section{References:}

1. Khera, D., John, J., Singh, K., \& Faruq, M. (2018). Tay-Sachs disease: a novel mutation from India. BMJ Case Reports, 11(1), e225916. doi:10.1136/bcr-2018225916

2. Sandhoff, K. (1969). Variation of $\beta$-N-acetylhexosaminidase-pattern in Tay-Sachs disease. FEBS letters, 4(4), 351-354.

3. Discussion. (1998). Biochemical Pharmacology, 56(4), 421-430. doi:10.1016/s00062952(98)00115-4

4. Kaback, M. (1993). Tay-Sachs Disease- Carrier Screening, Prenatal Diagnosis, and the Molecular Era. JAMA, 270(19), 2307. doi:10.1001/jama.1993.03510190063028

5. Christensen, C., \& Choy, F. (2017). A prospective treatment option for lysosomal storage diseases: CRISPR/Cas9 gene editing technology for mutation correction in induced pluripotent stem cells. Diseases, 5(1), 6. 